\title{
EFEITO DA ATIVIDADE FÍSICA MODERADA SOBRE NEURÔNIOS MIOENTÉRICOS NITRÉRGICOS DO ÍLEO DE RATOS DE MEIA IDADE
}

\author{
José Antônio de Almeida Major ${ }^{1}$ \\ Naianne Kelly Clebis ${ }^{2}$ \\ Renata de Britto Mari ${ }^{3}$ \\ Karina Martinez Gagliardo ${ }^{4}$ \\ Juliana Plácido Guimarães ${ }^{5}$ \\ Romeu Rodrigues de Souza ${ }^{6}$ \\ Sandra Regina Stabille \\ Nelson Sérgio de Oliveira Dias ${ }^{8}$ \\ Ricardo de Melo Germano ${ }^{9 *}$
}

MAJOR, J. A. de A.; CLEBIS, N. K.; MARI, R. de B.; GAGLIARDO, K. M.; GUIMARÃES, J. P.; SOUZA, R. R. de; STABILLE, S. R.; DIAS, N. S. de O.; GERMANO, R. de M. Efeito da atividade física moderada sobre neurônios mioentéricos nitrérgicos do íleo de ratos de meia idade. Arq. Ciênc. Vet. Zool. UNIPAR, Umuarama, v. 18, n. 3, p. 141-147, jul./set. 2015.

RESUMO: Poucos são os relatos a respeito do efeito da atividade física sobre o plexo mioentérico ao longo do envelhecimento. Portanto, analisaram-se os neurônios mioentéricos NADPH-diaforase positivos do íleo em ratos que envelheceram praticando atividade física a partir dos seis meses de vida. Dez ratos Wistar foram distribuídos em dois grupos ( $\mathrm{n}=5 / \mathrm{grupo}$ ): $\mathrm{S}$ (ratos sedentários controles com 12 meses de idade) e T (ratos treinados com 12 meses de idade). Os animais do grupo T realizaram, dos seis aos 12 meses de idade, atividade física regulada conforme resultados obtidos em teste de esforço máximo. Ao final do período experimental, o íleo obtido de cada animal foi processado para a técnica de NADPH-diaforase para evidenciar neurônios mioentéricos em preparados de membrana e quantificar e mensurar a área do pericário desses neurônios. A área do pericário não diferiu $(\mathrm{P}>0,05)$ entre o grupo $\mathrm{S}\left(218,49 \mu \mathrm{m}^{2}\right)$ e o grupo $\mathrm{T}\left(210,59 \mu \mathrm{m}^{2}\right)$. A média de neurônios presente em 60 campos microscópicos de preparados de membranas no grupo $\mathrm{S}(126$ neurônios) foi superior $(\mathrm{P}<0,05)$ a média encontrada no grupo T (93 neurônios), sugerindo que a atividade física pode inibir ou diminuir a expressão dos neurônios nitrérgicos em ratos de 12 meses de idade que praticaram atividade física desde os seis meses de vida. Os resultados quantitativos sugerem que a falta de atividade física regular propicia aumento na atividade dos neurônios nitrérgicos, contribuindo para o surgimento dos distúrbios da motricidade gastrointestinal que podem ocorrer ao longo do envelhecimento.

PALAVRAS-CHAVE: Corrida em esteira. Exercício físico. Intestino. NADPH-diaforase. Plexo mioentérico.

\section{THE EFFECT OF MODERATE PHYSICAL ACTIVITY ON MYOENTERIC NITRERGIC NEURONS IN THE ILEUM OF MIDDLE-AGED RATS}

\begin{abstract}
There are only a few reports on the effect of physical activity in the myoenteric plexus throughout the aging process. For such, the positive myoenteric NADPH-diaphoresis neurons in the ileum of those rats that aged practicing physical activity from the sixth month were analyzed. Ten Wistar rats were distributed into two groups ( $\mathrm{n}=5 /$ group): $\mathrm{S}$ (12 months-old control sedentary rats) and $\mathrm{T}$ (12 months-old trained rats). The animals from the T group practiced controlled physical activity from the age of 6 months to 12 months, according to the maximum effort test results. At the end of the experimental period, the ileum obtained from each animal was processed in the NADPH-diaphoresis technique to highlight myoenteric neurons in membrane preparations, and quantify and measure the perikaryon area from those neurons. The Perikaryon area did not differ $(\mathrm{P}>0.05)$ among the $\mathrm{S}$ group $\left(218.49 \mu \mathrm{m}^{2}\right)$ and the $\mathrm{T}$ group $\left(210.59 \mu \mathrm{m}^{2}\right)$. The average of neurons in 60 membrane preparations in the $\mathrm{S}$ group (126 neurons) was higher $(\mathrm{P}<0.05)$ than the average finding in the $\mathrm{T}$ group (93 neurons), indicating that the physical activity can inhibit or decrease the nitrergic expression in 12 month-old rats that have practiced physical activity from the age of 6 months. The quantitative results suggest that the lack of regular physical activity provides the elevation of nitrergic neurons, concurring to the emergence of gastrointestinal motility disorders that can occur during the aging process.
\end{abstract}

KEYWORDS: Intestine. Myoenteric Plexus. NADPH-diaphoresis. Physical activity. Treadmill Run.

\footnotetext{
DOI: https://doi.org/10.25110/arqvet.v18i3.2015.5531

${ }^{1}$ Bolsista PEBIC/CNPq - Universidade Paranaense - UNIPAR, Campus Paranavaí - zeantonio major@hotmail.com

${ }^{2}$ Professora do Departamento de Morfologia da Universidade Federal do Rio Grande do Norte - UFRN - Natal - Rio Grande do Norte - Brasil - nkclebis@ hotmail.com

${ }^{3}$ Professora do Departamento de Biologia Marinha da Universidade Estadual Paulista - UNESP - Campus Experimental do Litoral Paulista São Vicente, São Paulo, Brasil-remari@clp.unesp.br

${ }^{4}$ Professora da Universidade Monte Serrat - Unimonte - Santos, São Paulo, Brasil - karinamg79@uol.com.br

${ }^{5}$ Professora da Universidade Santa Cecília - Unisanta - Santos - São Paulo-Brasil - juvet@usp.br

${ }^{6}$ Professor do Curso de Pós-graduação em Anatomia dos Animais Domésticos e Silvestres da Faculdade de Medicina Veterinária e Zootecnia da Universidade de São Paulo - FMVZ-USP - São Paulo, São Paulo, Brasil - romeurodrigues@usp.br

${ }^{7}$ Professora do Departamento de Enfermagem da Faculdade Adventista Paranaense - FAP/IAP - Ivatuba - PR, Brasil - profasandra.regina@gmail.com ${ }^{8}$ Discente do Mestrado em Ciência Animal da Universidade Paranaense - UNIPAR, bolsista PIT/UNIPAR. nelsonsergiooliveira@gmail.com

${ }^{9}$ Professor do Mestrado em Ciência Animal da Universidade Paranaense - UNIPAR - Campus - Sede - Praça Mascarenhas de Moraes, 4282 - Zona III 87.502-210, Umuarama - PR, Brasil, prof.ricardogermano@gmail.com - * Autor para correspondência.
} 


\section{EFECTO DE LA ACTIVIDAD FÍSICA MODERADA EN NEURONAS MIENTÉRICAS NITRÉRGICAS DEL ÍLEON DE RATAS DE MEDIANA EDAD}

RESUMEN: Hay pocos informes sobre el efecto de la actividad física sobre el plexo mientérico a lo largo del envejecimiento. Por lo tanto, se analizaron las neuronas mientéricas NADPH-diaforasa positivas del íleon en ratas que envejecieron practicando actividad física a partir de seis meses de vida. Diez ratas Wistar se dividieron en dos grupos ( $\mathrm{n}=5$ / grupo): $\mathrm{S}$ (ratas sedentarias de control con 12 meses de edad) y T (ratas entrenadas con 12 meses de edad). Los animales del grupo T realizaron, de los seis a los doce meses de edad, actividad física regulada conforme resultados obtenidos en prueba de esfuerzo máximo. Al final del período experimental, el íleon obtenido de cada animal se procesó por la técnica de NADPH-diaforasa para mostrar las neuronas mientéricas en preparados de membrana, cuantificar y medir el área del pericario de esas neuronas. El área del pericario no difirió $(\mathrm{P}>0.05)$ entre el grupo $\mathrm{S}(218.49 \mu \mathrm{m} 2)$ y el grupo $\mathrm{T}(210.59 \mu \mathrm{m} 2)$. El número medio de neuronas presentes en 60 campos microscópicos de preparados de membranas en el grupo $\mathrm{S}$ (126 neuronas) fue mayor ( $\mathrm{P}$ $<0,05)$, promedio encontrado en el grupo $\mathrm{T}$ (93 neuronas), lo que sugiere que la actividad física puede inhibir o disminuir la expresión de las neuronas nitrérgicas en ratas de 12 meses de edad que practicaron actividad física desde los seis meses de edad. Los resultados cuantitativos sugieren que la falta de actividad física regular promueve aumento en la actividad de las neuronas nitrérgicas, contribuyendo a la aparición de disturbios de la motricidad gastrointestinal que pueden ocurrir a lo largo del envejecimiento.

PALABRAS CLAVE: Carrera en esterilla. Ejercicio físico. Intestino. NADPH-diaforasa. Plexo mientérico.

\section{Introdução}

O sistema nervoso entérico (SNE) pode ser considerado um sistema independente que controla e coordena a motilidade, o fluxo sanguíneo e as secreções necessárias para a digestão (GABELLA, 1979; SCHEMANN; NEULIST, 2004).

Entre os componentes do SNE encontram-se os plexos mioentérico e submucoso. O plexo mioentérico está localizado na túnica muscular do tubo digestório e contém uma quantidade variável de diferentes tipos de neurônios classificados e identificados segundo suas características morfológicas, elétricas, neuroquímicas e funcionais (BREHMER; SCHRODL; NEUHUBER, 1999; FURNESS, 2000; FURNESS, 2006; GABELLA, 1979; GALLIGAN et al., 2000; LOMAX; FURNESS, 2000; SCHEMANN; NEUNLIST, 2004).

A população total de neurônios mioentéricos e respectivas subpopulações podem ser evidenciadas por diferentes métodos que incluem desde a impregnação por prata, como as colorações com azul de metileno, as técnicas histoquímicas e imunohistoquímicas (GABELLA, 1969; MARESE; FREITAS; NATALI, 2007; SANTER, 1994; ZANONI et al., 2005).

Entre os tipos de neurônios do plexo mioentérico estão aqueles que expressam o neurotransmissor óxido nítrico que é inibitório da musculatura circular e promove o relaxamento da musculatura gastrointestinal (VAN GELDREE; LEFEBVRE, 2004).

O efeito do envelhecimento sobre o tubo gastrointestinal e sobre o plexo mioentérico tem sido estudado (COWEN et al., 2000; MARESE; FREITAS; NATALI, 2007; PHILLIPS et al., 2007; PHILLIPS; POWLEY, 2001, 2007; WADE; EVANS; LIEB, 2002;).

Com o avançar da idade, ocorre diminuição na motilidade esofágica e colônica (BUTT et al., 1993; HOLLINS; CASTELL, 1974; KHAN et al., 1977; WADE; EVANS; LIEB, 2002), comprometimento do esvaziamento gástrico (BROGNA et al., 1999; HOROWITZ et al., 1984; SMITS; LEFEBVRE, 1996) e aumento no trânsito intestinal (ANURAS; LEONING-BAUCKE, 1984; WADE; EVANS; LIEB, 2002). A alteração do número de neurônios mioentéricos é um dos fatores que contribuem para o comprometimento da motilidade do tubo digestório ao longo do envelhecimento (DE SOUZA et al., 1993; EL-SALHY; SABDSTRÖM; HOLMLUND, 1999; GABELLA, 1989; GOMES; DE SOUZA; LIBERTI, 1997; PHILLIPS; POWLEY, 2001, 2007; SANTER; BAKER, 1988).

As alterações constatadas com a senilidade no tubo digestório correlacionam-se também com a deterioração da função autonômica. Acredita-se que a atividade física poderia ter alguma influência protetora sobre o tubo gastrointestinal (DAPOIGNY; SARNA, 1991). Contudo, os resultados das pesquisas ainda não são conclusivos (CAMMACK et al., 1982; FORDTRAN; SALTIN, 1967; MOORE; DATZ; CHRISTIAN, 1990; ROBERTSON et al., 1993).

De maneira geral, o treinamento físico diminui a atividade simpática (KRIEGER; BRUM; NEGRÃO, 1999; LIU; KULAKOFSKY; ZUCKER, 2002) e aumenta o tônus parassimpático nos intervalos e durante o exercício (KRIEGER; BRUM; NEGRÃO, 1999; LIU; KULAKOFSKY; ZUCKER, 2002; NEGRÃO et al., 1992). Além disso, no hipocampo foi verificada neurogênese associada à atividade física (ARIDA et al., 2004; VAN PRAAG et al., 1999).

Dessa forma, considerando que a atividade física pode contribuir para atenuar o efeito do envelhecimento sobre o SNE, objetivou-se analisara densidade e a dimensão da área do pericário de neurônios mioentéricos NADPH-diaforase positivos (NADPH-dp) do íleo de ratos que envelheceram realizando exercício físico regular (corrida em esteira).

\section{Material e Métodos}

Esse estudo foi conduzido de acordo com os princípios éticos do Colégio Brasileiro de Experimentação Animal (COBEA) e foi aprovado pela Comissão de Bioética da Faculdade de Medicina Veterinária e Zootecnia da Universidade de São Paulo (FMVZ-USP), protocolo no 734/2005.

Foram utilizados dez ratos machos (Rattus norvegicus), da linhagem Wistar, inicialmente com seis meses de idade, provenientes do Biotério Central da Universidade Federal de São Paulo (UNIFESP). Os animais provenientes de um protocolo experimental de exercício físico foram distribuídos em dois grupos $(\mathrm{n}=5 /$ grupo): sedentário $(\mathrm{S})$ e trei- 
nado (T).

Os animais permaneceram alojados em caixas de polipropileno providas de bebedouro e comedouro, e mantidas em condições ambientais controladas de temperatura $\left(24 \pm 2^{\circ} \mathrm{C}\right)$ e de iluminação (ciclo de 12 horas claro/12 horas escuro) recebendo ração $\left(\right.$ Nuvital $\left.^{\circledR 10}\right)$ e água ad libitum. Os animais foram pesados mensalmente.

\section{Protocolo experimental (exercício físico)}

Os animais foram submetidos a duas semanas de adaptação ao exercício físico (corrida em esteira) e, após este período, os grupos foram separados de forma homogênea. Somente os animais que possuíam a habilidade de correr foram mantidos no experimento segundo a classificação de Dishman et al. (1988).

Os animais dos grupos $\mathrm{S}$ e $\mathrm{T}$ realizaram teste de esforço máximo (TEM) em esteira elétrica a velocidade de $0,3 \mathrm{Km} / \mathrm{h}$ (ou $5 \mathrm{~m} / \mathrm{min}$ ). A cada quatro minutos, a velocidade da esteira foi aumentada na mesma proporção $(0,3 \mathrm{Km} / \mathrm{h})$ conforme protocolo de Silva et al. (1997).

Os animais do grupo $\mathrm{T}$ foram então submetidos a seis meses de treinamento em esteira, cinco dias por semana, com carga progressiva até $60 \%$ daquela conseguida pelo grupo no teste de esforço. O tempo de atividade física variou de 30 a 60 minutos. Os TEMs foram realizados a cada quatro semanas, totalizando sete testes ao final do experimento.

Os animais do grupo $\mathrm{S}$ realizaram atividade física apenas uma vez por semana à velocidade de $0,3 \mathrm{Km} / \mathrm{h}$, pelo período de dez minutos, apenas para manterem a habilidade de correr na esteira.

\section{Obtenção do íleo}

Ao final do experimento, os animais, então com 12 meses de idade, receberam dose de anestésico tiopental (40mg/Kg de peso corpóreo) por via intravenosa (veia peniana). Em seguida, foram laparotomizados por meio de incisão ventral mediana e, para cada animal, o íleo foi isolado tendo como referência a prega íleo-cecal (limite cranial) e a junção íleo-cecal (limite caudal).

\section{Histoquímica de NADPH-diaforase (SCHERER-SIN- GLER et al., 1983)}

O íleo obtido de cada animal foi lavado e preenchido com tampão fosfato de sódio (PBS) em pH 7,4. Para manter o preenchimento, as extremidades do íleo foram atadas com fio de sutura.

Em seguida, para evidenciar os neurônios, cada íleo foi submetido a duas lavagens em PBS (10 minutos cada) seguidas de permeabilização em PBS contendo Triton X-100 $\left(\right.$ Sigma $\left.^{\circledR 11}\right)$ a $0,3 \%$ dissolvido em tampão fosfato de sódio $(\mathrm{pH} 7,3)\left(\right.$ Sigma $\left.^{\circledR}\right)$ durante dez minutos. Depois, mais duas lavagens em PBS (10 minutos cada).

Para visualizar os neurônios mioentéricos NADPH-diaforase positivos (neurônios NADPH-dp) cada íleo foi incubado em meio de reação constituído por $50 \mathrm{mg}$ de NBT

\footnotetext{
${ }^{10}$ Ração Nuvital - Nuvital Nutrientes, Colombo - PR.

${ }^{11}$ Sigma-Aldrich Corporation - St. Louis - Missouri - United States of America.
}

$\left(\right.$ Sigma $\left.^{\circledR}\right), 100 \mathrm{mg}$ de $\beta$-NADPH $\left(\right.$ Sigma $\left.^{\circledR}\right)$ e Triton X-100 ${ }^{\circledR}$ $0,3 \%\left(\right.$ Sigma $\left.^{\circledR}\right)$ em tampão Tris-HCl $\left(\operatorname{Sigma}^{\circledR}\right)(0,1 \mathrm{M}, \mathrm{pH}$ $7,6)$ por duas horas.

Após, as extremidades do íleo foram liberadas das suturas e o íleo foi imerso em solução de paraformaldeído a $4 \%$ para a fixação e estocagem.

\section{Obtenção dos preparados de membrana}

Cada íleo foi seccionado longitudinalmente ao longo de sua borda mesentérica. Ao estereomicroscópio, a mucosa e a submucosa foram retiradas e desprezadas e as túnicas muscular e serosa preservadas constituíram o preparado de membrana.

Os preparados de membrana obtidos foram desidratados em série crescente de álcoois $(90 \%, 95 \%$ e absoluto), diafanizados em três imersões consecutivas de xilol e colocados entre lâmina e lamínula de vidro com glicerina $\left(\operatorname{Synth}^{\circledR 12}\right)$ tamponada em tampão fosfato de sódio $\left(\operatorname{Sigma}^{\circledR}\right)$.

\section{Análise quantitativa}

Para a quantificação neuronal, o preparado de membrana obtido do íleo de cada animal foi visualizado ao microscópio de luz (Olympus-BX 40). A imagem verificada em cada campo do microscópio foi capturada por câmera digital de alta resolução AxioCam HRc - Zeiss ${ }^{\circledR 13}$ e transferida para o computador para quantificação dos neurônios.

Em cada preparado de membrana, os neurônios foram quantificados em imagens de 60 campos microscópicos que amostraram igualmente toda a extensão da circunferência do íleo. A área de cada campo microscópico era 0,086 $\mathrm{mm}^{2}$, sendo que os 60 campos representaram a área de 5,16 $\mathrm{mm}^{2}$ de íleo.

\section{Morfometria da área do pericário dos neurônios}

A mensuração da área do pericário dos neurônios mioentéricos NADPH-dp foi realizada pelo programa de análise de imagem computadorizado Image Pro Plus 3.0.1 ${ }^{\circledR 14}$.

Para tanto, as imagens dos neurônios capturadas por câmera digital para a quantificação neuronal foram utilizadas.

Foi mensurada a área do pericário $\left(\mathrm{em} \mu \mathrm{m}^{2}\right)$ de todos os neurônios quantificados em cada preparado de membrana.

\section{Análise estatística}

Os resultados referentes à quantificação neuronal foram analisados pelo teste Kruskal-Wallis para comparação entre os grupos. Já os resultados obtidos na morfometria da área do pericário dos neurônios foram comparados pelo teste T de Student. Para todos os testes, o nível de significância adotado foi $5 \%$.

A densidade neuronal por $5,16 \mathrm{~mm}^{2}$ de íleo e os dados obtidos na mensuração da área do pericário foram ex-

${ }^{12}$ Synth - Produtos Químicos, Reagente Analíticos e Matérias-Primas - Diadema - SP.

${ }^{13}$ Zeiss - Thornwood, New York - United States of America.

${ }^{14}$ Media Cybernetics, Silver Spring, Maryland, United States of America. 
pressos como média \pm desvio padrão da média.

\section{Resultados}

O peso corporal inicial dos animais do grupo $\mathrm{S}$ e do grupo $\mathrm{T}$ não diferiu ( $\mathrm{P}>0,05)$, o mesmo ocorrendo com o peso final (Tabela 1). A média do peso final nos animais do grupo $\mathrm{T}$ foi aproximadamente $17 \%$ menor do que a dos animais do grupo $\mathrm{S}$, porém não alcançou diferença estatística significativa.

Os neurônios mioentéricos NADPH-dp de ambos os grupos estudados foram localizados na túnica muscular, organizados predominantemente em gânglios interconectados por uma rede de fibras nervosas (Figura 1). Neurônios isolados, especialmente no trajeto das fibras nervosas, foram também encontrados. A organização do plexo na túnica muscular não diferiu entre os grupos $\mathrm{S}$ e T.

Figura 1: Fotomicrografia de preparado de membrana com gânglios do plexo mioentérico do íleo dos ratos dos grupos sedentário (S) e treinado $(\mathrm{T})$, evidenciando os neurônios NADPH-diaforase positivos (seta) organizados predominantemente no interior dos gânglios. (Barra: $100 \mu \mathrm{m}$ ).

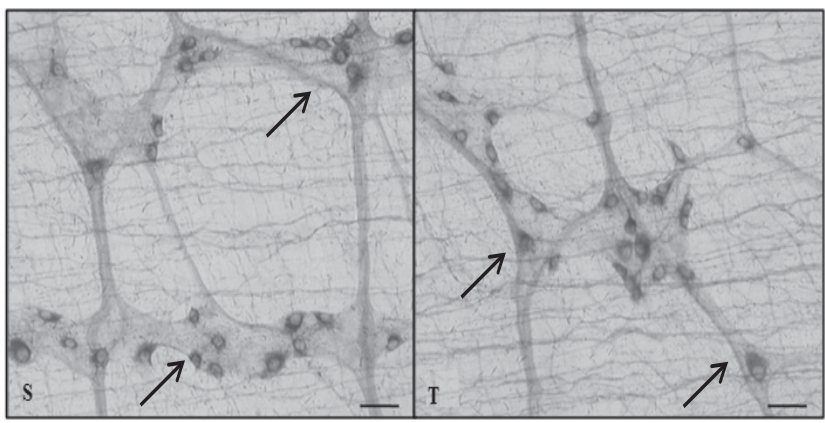

Quantificação neuronal e mensuração da área do pericário

Os resultados referentes às análises quantitativa e morfométrica dos neurônios NADPH-dp encontram-se na Tabela 1. A média de neurônios mioenwtéricos presente em 60 campos microscópicos $\left(5,16 \mathrm{~mm}^{2}\right.$ de íleo) do íleo dos animais do grupo $\mathrm{S}(126 \pm 6,87$ neurônios $)$ foi superior $(\mathrm{P}<0,05)$ à média encontrada nos animais do grupo $\mathrm{T}(93 \pm 16,8$ neurônios).

Tabela 1: Média e desvio padrão dos pesos corporais inicial e final, da densidade de neurônios e da área do pericário de neurônios NADPH-diaforase positivos (NADPH-dp) presentes em 60 campos microscópicos de preparado de membrana do íleo dos ratos dos grupos sedentário $(\mathrm{S})$ e treinado $(\mathrm{T})$.

\begin{tabular}{ccccc}
\hline Grupos & $\begin{array}{c}\text { Peso inicial } \\
(\mathrm{g})\end{array}$ & Peso final $(\mathrm{g})$ & $\begin{array}{c}\text { Densidade neuronal } \\
\text { NADPH-dp }\end{array}$ & $\begin{array}{c}\text { Área do pericário }\left(\mu \mathrm{m}^{2}\right) \text { dos } \\
\text { neurônios NADPH-dp }\end{array}$ \\
\hline $\mathbf{S}(\mathbf{n}=\mathbf{5})$ & $421 \pm 9,1^{\mathrm{a}}$ & $488 \pm 11,64^{\mathrm{b}}$ & $126 \pm 6,87^{\mathrm{a}}$ & $218,49 \pm 64,28^{\mathrm{a}}$ \\
$\mathbf{T}(\mathbf{n}=\mathbf{5})$ & $421,3 \pm 7,9^{\mathrm{a}}$ & $468,5 \pm 9,9^{\mathrm{b}}$ & $93 \pm 16,79^{\mathrm{b}}$ & $210,59 \pm 63,78^{\mathrm{a}}$ \\
\hline
\end{tabular}

* Médias seguidas por letras diferentes na mesma coluna diferem $(\mathrm{P}<0,05)$ pelo teste de Kruskal-Wallis no caso da densidade neuronal e pelo teste T de Student para o peso dos ratos e morfometria neuronal.

Não houve diferença significativa $(\mathrm{P}>0,05)$ entre as médias da área do pericário dos neurônios entre o grupo $\mathrm{S}$ $\left(218,49 \pm 64,28 \mu \mathrm{m}^{2}\right)$ e o grupo T $\left(210,59 \pm 63,78 \mu \mathrm{m}^{2}\right)$. Em ambos os grupos predominaram neurônios com área do pericário entre 200 e $300 \mu \mathrm{m}^{2}$.

\section{Discussão}

Em relação ao peso dos animais, constatou-se ao final do período experimental que as médias do peso corporal não diferiram $(\mathrm{P}>0,05)$ entre os grupos $\mathrm{S}$ e T. Embora sem significância estatística, o peso dos animais do grupo $\mathrm{T}$ foi $4 \%$ inferior ao do grupo $\mathrm{S}$. Menor peso corporal de indivíduos treinados em relação a indivíduos sedentários foi verificado em humanos (KYLE et al., 2004). Os indivíduos que participam de um programa de treinamento aumentam o seu peso corporal magro e reduzem a gordura corporal. Contudo, dependendo do grau de envelhecimento, ocorre perda de peso dado o declínio geral na função neuromuscular e a deterioração na capacidade celular de realizar a síntese proteica (MCARDLE; KATCH; KATC, 1998).

Estudos recentes da morfologia da parede intestinal têm demonstrado que a prática habitual de atividade física moderada protege a parede do íleo de camundongos contra os efeitos do envelhecimento (ROSA et al., 2005). Entretanto, as ações da atividade física sobre os neurônios do SNE ainda são pouco discutidas e conhecidas.

Em relação à organização espacial dos neurônios mioentéricos NADPH-dp na túnica muscular do íleo, não se observaram diferenças entre os grupos estudados (grupos $\mathrm{S}$ e T). Os neurônios mioentéricos foram localizados na túnica muscular, organizados predominantemente em gânglios interconectados por fibras nervosas como descrito para o plexo mioentérico de roedores e outras espécies animais (GABELLA, 1979, MIRANDA-NETO et al., 2001; MOLINARI et al., 1994; SANT'ANA et al., 1997).

A histoquímica da NADPH-diaforase utilizada neste estudo evidencia os neurônios que sintetizam óxido nítrico (NO), um neurotransmissor inibitório da musculatura intestinal (FURNESS, 2000, 2006; SANDERS; WARD, 1992). Os neurônios que expressam NO têm sido identificados também por meio de técnicas imunohistoquímicas para marcação da enzima óxido nítrico sintase, sendo que a distribuição da atividade NADPH-diaforase nos neurônios entéricos tem-se mostrado idêntica a imunorreatividade a óxido nítrico sintase (FURNESS et al., 1994; BELAI; COOPER; BURNSTOCK, 1995; SAFFREY, 2004).

A densidade dos neurônios NADPH-dp diferiu $(\mathrm{P}<0,05)$ entre os grupos. $\mathrm{O}$ grupo $\mathrm{T}$ apresentou média de neurônios inferior ao do grupo S. Do mesmo modo, Gagliardo et al. (2008) relataram diminuição no número de neurônios NADPH-dp no colo de ratos que, dos seis aos 12 meses de idade, foram submetidos ao exercício físico regular em esteira. Por outro lado, Silva Porto et al. (2012) descreveram diminuição no número total de neurônios mioentéricos e aumento na quantidade de neurônios mioentéricos NADPH-dp no duodeno de ratos de 18 meses de idade quando comparados com ratos mais jovens (seis meses de idade). Essas pesquisas indicam que a atividade de neurônios mioentéricos NADPH-dp aumenta com a idade do animal, contribuindo para o surgimento de distúrbios gastrointestinais.

Assim, a densidade neuronal menor para os ratos do 
grupo $\mathrm{T}$ constatada no presente trabalho sugere que a atividade física pode postergar os efeitos do envelhecimento nos neurônios miontéricos do íleo de ratos.

De maneira geral, a atividade dos neurônios simpáticos após a atividade física é diminuída (KRIEGER; BRUM; NEGRÃO, 1999; LIU; KULAKOFSKY; ZUCKER, 2002), contribuindo para estimular o peristaltismo intestinal. Alguns estudos têm mostrado relação inversa entre exercício físico e constipação (EVERHART et al., 1989; KINNUNEN, 1991; SULLIVAN; WONG, 1992). Assim, a atividade física moderada também poderia inibir os neurônios mioentéricos nitrérgicos, diminuindo a síntese e expressão de NO nesses neurônios e tornando-os menos reativos à histoquímica da NADPH-diaforase, justificando a redução de aproximadamente $26 \%$ na densidade neuronal nos animais do grupo $\mathrm{T}$ quando comparada ao do grupo $\mathrm{S}$.

Entre os neurônios NADPH-dp dos grupos $\mathrm{S}$ e T predominaram neurônios com área do pericário entre $200 \mathrm{e}$ $300 \mathrm{~m}^{2}$. A média da área do pericário dos neurônios não diferiu $(\mathrm{P}>0,05)$ entre os grupos $\mathrm{T}\left(210,59 \pm 63,78 \mu \mathrm{m}^{2}\right)$ e $\mathrm{S}$ $\left(218,49 \pm 64,28 \mu \mathrm{m}^{2}\right)$. Resultados semelhantes foram descritos para os neurônios mioentéricos NADH-dp do duodeno de ratos submetidos às mesmas condições experimentais (MARI et al., 2008). Silva Porto et al. (2012) e Marese et al. (2007) também não constataram diferenças entre a área do pericário de neurônios NADPH-dp e de neurônios imunomarcados para Miosina- $\mathrm{V}$ no duodeno de ratos ao longo do envelhecimento, respectivamente.

\section{Conclusões}

A prática de atividade física regular dos seis aos 12 meses de idade pode inibir ou diminuir a atividade dos neurônios nitrérgicos em ratos, contribuindo para retardar ou minimizar o surgimento de distúrbios da motricidade intestinal característicos do envelhecimento. O sedentarismo contribui para maior atividade de neurônios NADPH-dp, propiciando o surgimento de distúrbios gastrointestinais.

\section{Agradecimentos}

Os autores agradecem ao $\mathrm{CNPq}$ pela concessão da bolsa PEBIC para o primeiro autor, e a UNIPAR pelo suporte financeiro para o desenvolvimento da Iniciação Científica.

\section{Referências}

ANURAS, S., LEONING-BAUCKE, V. Gastrointestinal motility in the elderly. Journal of the American Geriatrics Society, New York, v. 32, n. 5, p.386-390. 1984

ARIDA, R. M. et al. Differential effects of the spontaneous versus forced exercise in rats on the staining of parvalbumin-positive neurons in the hippocampal formation. Neuroscience Letters, Amsterdam, v. 364, n. 3 , p. $135-138,2004$

BELAI, A.; COOPER, S.; BURNSTOCK, G. Effect of age on NADPH-diaforase-containing myenteric neurones of rat ileum and proximal colon. Cell \& Tissue Research, Berlin, v. 279 , p. $379-383,1995$
BREHMER, A.; SCHRODL, F.; NEUHUBER, W.

Morphological classifications of enteric neurons - 100 years after Dogiel. Anatomy and Embryology, Berlin, v. 200, p. 125-135, 1999.

BROGNA, A. et al. Influence of aging on gastrointestinal transit time: An Ultrasonographic and Radiologic Study. Investigative Radiology, Philadelphia, v. 34, n. 5, p. $357-$ 359, 1999.

BUTT, W.G. et al. Age-related changes in rat colon mechanics. Neurogastroenterology \& Motility, Osney Mead, v. 5, n. 2, p. 123-128, 1993.

CAMMACK, J. et al. Effect of prolonged exercise on the passage of a solid meal through the stomach and small intestine. Gut, London, v. 23, n. 11, p. 957-961, 1982.

COWEN, T. et al. Restricted diet rescues rat enteric motor neurons from age related cell death. Gut, London, v. 47, n. 5, p. 653-660, 2000.

DAPOIGNY, M., SARNA, S. K. Effects of physical exercise on colonic motor activity. The American Journal of Physiology, Washington, v. 260, n. 4, p. G646-G652, 1991.

DE SOUZA, R. R. et al. Age-induced nerve cell loss in the myenteric plexus of the small intestine in man. Gerontology, Basel, v. 39, n. 4, p. 183-188, 1993.

DISHMAN, R. K. et al. Open-field behavior is not related to treadmill performance in exercising rats. Physiology $\&$ Behavior, Oxford, v. 43, n. 5, p. 541-546. 1988.

EL-SALHY, M.; SABDSTRÖM, O.; HOLMLUND, F. Ageinduced changes in the enteric nervous system in the mouse. Mechanisms of Ageing and Development, Lausanne, v. 107, n. 1, p. 93-103, 1999.

EVERHART, J. E. et al. A longitudinal survey of selfreported bowel habits in the United States. Digestive Diseases and Sciences, New York, v. 34, n. 8, p. 1153-62, 1989.

FORDTRAN, J. S.; SALTIN, B. Gastric emptying and intestinal absorption during prolonged severe exercise. Journal of Applied Physiology, Washington, v. 23, n. 3, p. 331-335, 1967.

FURNESS, J. B. The enteric nervous system. Oxford: Blackwell Publishing. 2006. 274 p.

FURNESS, J. B. Types of neurons in the enteric nervous system. Journal of the Autonomic Nervous System, Amsterdam, v. 81, n. 1-3, p. 87-96, 2000.

GABELLA, G. Detection of nerve cells by a histochemical technique. Experientia, Basel, v. 25, n. 2, p. 218-219, 1969.

GABELLA, G. Innervation of gastrointestinal tract. 
International Review of Cytology, New York, v. 79, p. 129-193, 1979.

GABELLA, G. Fall in the number of myenteric neurons in aging guinea pigs. Gastroenterology, Baltimore, v. 96, n. 6, p. 1487-1493, 1989.

GAGLIARDO, K. M. et al. Exercise reduces inhibitory neuroactivity and protects myenteric neurons from agerelated neurodegeneration. Autonomic Neuroscience: basic \& clinical, Amsterdam, v. 141, n. 1-2, p. 31-37, 2008.

GALLIGAN, J. J. et al. Multiple mechanisms of fast excitatory synaptic transmission in the enteric nervous system. Journal of the Autonomic Nervous System, Amsterdam, v. 81, n. 1-3, p. 97-103, 2000.

GOMES, O. A.; DE SOUZA, R. R.; LIBERTI, E. A. A preliminary investigation of the effects of ageing on the nerve cell number in the myenteric ganglia of the human colon. Journal of the Autonomic Nervous System, Basel, v. 43, n. 4, p. 210-217, 1997.

HOLLINS, J. B.; CASTELL, D. O. Esophageal function in elderly men: a new look at presbuyoesophagus. Annals of Internal Medicine, Philadelphia, v. 80, n. 3, p. 371. 1974.

HOROWITZ, M. et al. Changes in gastric emptying rates with age. Clinical Science, London v. 67, n. 2, p. 213-218, 1984.

KHAN, T. A. et al. Esophageal motility in the elderly. Digestive DIseases and Sciences, New York, v. 22, n. 12, p. 1049, 1977.

KINNUNEN, O. Study of constipation in a geriatric hospital, day hospital, old people's home and at home. Aging Cell, Oxford, v. 3, n.2, p. 161-70, 1991.

KRIEGER, E. M.; BRUM, P. C.; NEGRÃO, C. E. Influence of exercise training on neurogenic control of blood pressure in spontaneously hypertensive rats. Hypertension, Dallas, v. 34, p. 720-723, 1999.

KYLE, G. T. et al. An examination of recreationists relationship with activities and settings. Leisure Science, New York, v. 26, n. 2, p. 123-141, 2004.

LIU, J.; KULAKOFSKY, J.; ZUCKER, I. H. Exercise training enhances baroreflex control of heart rate by a vagal mechanism in rabbits with heart failure. Journal of Applied Physiology, Washington, v. 92, n. 6, p. 2403-2408, 2002.

LOMAX, A. E.; FURNESS, J. B. Neurochemical classification of enteric neurons in the guinea-pig distal colon. Cell \& Tissue Research, Berlin, v. 302, n. 1, p. 5972,2000

MCARDLE, W. D.; KATCH, F. I.; KATCH, V. L. Fisiologia do exercício. Energia, nutrição e desempenho humano. 6 ed. Rio de Janeiro: Guanabara Koogan. 2008.
1099 p.

MARESE, A. C. M.; FREITAS, P.; NATALI, M. R. M. Alterations of the number and profile of myenteric neurons of Wistar rats promoted by age. Autonomic Neuroscience: basic \& clinical, Amsterdam, v. 137, n. 1, p. 10-18, 2007.

MARI, R. B. et al. Effects of exercise on the morphology of the myenteric neurons of the duodenum of Wistar rats during the ageing process. Anatomia, histologia, embryologia, Berlin, v. 37, n.4, p. 289-295, 2008.

MIRANDA-NETO, M. H. et al. Regional diferences in the number and type of myenteric neurons of the ileum of rats. Arquivos de Neuro-psiquiatria, São Paulo, v. 59, n. 1, p. 54-59, 2001.

MOLINARI, S. L. et al. Estudo morfológico do plexo mioentérico do estômago glandular do pato (Anas sp). Revista Unimar, Marília, v. 16, n. 2, p. 419-426, 1994.

MOORE, J. G.; DATZ, F. L.; CHRISTIAN, P. E. Exercise increases solid meal gastric emptying rates in men.

Digestive Diseases and Sciences, New York, v. 35, n. 4, p. 428-432, 1990.

NEGRÃO, C. E. et al. Vagal function impairment after exercise training. Journal of Applied Physiology, Washington, v. 72, n. 5, p. 1749-1753, 1992.

PHILLIPS, R. J.; POWLEY, T. L. As the gut ages: Timetables for aging of innervation vary by organ in the Fischer 344 rat. The Journal of Comparative Neurology, Philadelphia, v. 434, n. 3, p. 358-377, 2001.

PHILLIPS, R. J.; POWLEY, T. L. Innervation of the gastrointestinal tract: Patterns of aging. Autonomic Neuroscience: basic \& clinical, Amsterdam, v. 136, n 1-2, p. 1-19, 2007.

ROBERTSON, G. et al. Effects of exercise on total and segmental colon transit. Journal of Clinical Gastroenterology, New York, v. 16, n. 4, p. 300-303, 1993.

ROSA, E. F. et al. Habitual exercise program protects murine intestinal, skeletal, and cardiac muscles against aging. Journal of Applied Physiology, Washington, v. 99, n. 4 , p. $1569-75,2005$.

SAFFREY, M. J. Ageing of the enteric nervous system. Mechanisms of ageing and development, Lausanne, $\mathrm{v}$. 125, n.12, p. 899-906, 2004.

SANDERS, K. M.; WARD, S. M. Nitric oxide as a mediator of non adrenergic non cholinergic neurotransmission. The American Journal of Physiology, Washington, v. 262, n. 3, p. G379-G392, 1992.

SANT'ANA, D. M. G. et al. Neurons number in the myenteric plexus of the ascending colon of rats. A comparative study using two stainning techniques. 
Arquivos de Neuro-psiquiatria, São Paulo, v. 55, n. 3, p. 460-465, 1997.

SANTER, R. M. Survival of the population of NADPHdiaphorase stained myenteric neurons in the small intestine of aged rats. Journal of the Autonomic Nervous System, Amsterdam, v. 49, n. 2, p. 115-121, 1994.

SANTER, R. M.; BAKER, D. M. Enteric neuron numbers and sizez in Auerbach's plexus in the small and large intestine of adult and aged rats. Journal of the Autonomic Nervous System, Amsterdam, v. 25, n. 1, p. 59-67, 1988.

SCHEMANN, M.; NEUNLIST, M. The human enteric nervous system. Neurogastroenterology \& Motility, Osney Mead, v. 16, n. s1, p. 55-59. 2004.

SCHERER-SINGLER, U. et al. Demonstration of unique population of neurons with NADPHdiaphorasehistochemistry. Journal of Neuroscience Methods, Amsterdam, v. 9, n. 3, 229-234, 1983.

SILVA, G. J. J. et al. Acute and chronic effects of exercise on baroreflexes in spontaneously hypertensives. Hypertension, Dallas, v. 30, n. 3, p. 714-719, 1997.

SILVA PORTO, G. et al. Effect of caloric restriction on myenteric neuroplasticity in the rat duodenum during aging. Autonomic Neuroscience: basic \& clinical, Amsterdam, v. 168, n. 1, p. 43-47, 2012.

SMITS, G. J. M.; LEFEBVRE, R. A. Influence of aging on gastric emptying of liquids, small intestine transit, and fecal output in rats. Experimental Gerontology, Oxford, v. 31, n. 5, p. 589-596, 1996.

SULLIVAN, S. N.; WONG, C. Runners' diarrhea. Different patterns and associated factors. Journal of Clinical Gastroenterology, New York, v. 14, n. 2, p. 101-4, 1992.

VAN GELDRE, L. A. V.; LEFEBVRE, R. A. Interaction of NO and VIP in gastrointestinal smooth muscle relaxation. Current Pharmaceutical Design, Schiphol, v. 10, n. 20, p. 2483-2497, 2004.

VAN PRAAG, H. et al. Running enhances neurogenesis, learning, and long-term potentiation in mice. Proceedings of the National Academy of Sciences of the United States of America, Washington, v. 96, n. 23, p. 13427-13431, 1999.

WADE, P. R.; EVANS, B.; LIEB, J. Age-related changes in submucosal neurons and motility in Fischer 344 rat distal colon. In: Gastroenterology. INDEPENDENCE SQUARE WEST CURTIS CENTER, STE 300, PHILADELPHIA, PA 19106-3399 USA: WB SAUNDERS CO, 2002. p. A20-A20.

ZANONI, J. N. et al. Effects of supplementation with ascorbic acid for a period of 120 days on the myosin- $\mathrm{V}$ and NADPHd positive myenteric neurons of the ileum of rats.
Anatomia, Histologia, Embryologia, Berlin, v. 34, n. 3, p. 149-153, 2005.

Recebido em: 10.08.2015

Aceito em: 11.10.2015. 